\title{
Status of key faunal species in Koshi Tappu Wildlife Reserve after Koshi flood disaster 2008
}

\author{
Kul Prasad Limbu* and Bharat Raj Subba** \\ Department of Zoology, Post Graduate Campus, Biratnagar, Nepal, PO Box-137 \\ E-mail:*limbukp@gmail.com,**subbabharat@yahoo.com
}

\begin{abstract}
Present survey was carried out from April $16^{\text {th }}$ to December $15^{\text {th }}$ of 2009 with a view to assess the key faunal species of Koshi Tappu Wildlife Reserve such as Birds, Gangetic Dolphin, Wild water buffalo, different species of fish and their habitats after Koshi flood disaster 2008. Several trips were made within the KTWR using boat for surveying dolphin census, to make checklist of birds and fishes and to study wild water buffalo status. Among 120 species of birds belonging to 44 families and 103 genera were sighted, 27 species were found as winter visitor, 4 species as summer visitor and 89 species as resident birds. The census of Gangetic Dolphin was conducted in the Koshi river course starting from Rajabas to Koshi Barrage $(36 \mathrm{~km})$ in November-December 2009. The census concluded with a sighting of 6 dolphins in the river section north of Koshi Barrage upto Rajabas and 5 dolphins in the downstream of Koshi Barrage. The status of wild water buffalo was also studied in different areas of KTWR. Regular collection of fish enlisted 64 species belonging to 15 families and 40 genera.
\end{abstract}

Key words: Key faunal species, Koshi Tappu Wildlife Reserve, flood disaster

\section{Introduction}

As a need was felt to conserve the endangered wild water buffaloes and their habitat, Koshi Tappu Wildlife Reserve (KTWR) was established in July 1976 over 6500 ha under the National Parks and Wildlife Conservation Act 1973 and extended in 1980 including the flood plains of the Koshi river. Koshi Tappu Wildlife Reserve lies in the flood plain area, formed as a result of braiding and meandering of the Saptakoshi river, one of the major tributaries of Ganges, originating from Tibetan plateau and the snow peaks in the Himalayas. It is high-ranked silt carrying river in the world (Sah, 1997). It drains a total area of $69,300 \mathrm{~km}^{2}$ up to its confluence with the Ganges in India. Because of high siltation rate in the river, frequent changes of the river course takes place every year and sometimes shifts position from one side of the floodplain to the other. As silt is deposited, new sandy islands are formed each year. Before construction of the barrage and the embankment, the Koshi river had spread hundreds of kilometers east and west in Nepal as well as in India. After the construction of embankments and barrage, the river had been controlled till last the 18th August 2008, when the river showed its past behaviour again by devastating the embankment and changed its course. The Koshi river breached the nose of spur 12.90 and 12.10 on the eastern embankment, $12 \mathrm{~km}$ north of the barrage affecting six village development committees (VDC) of Sunsari district in Nepal and 14 districts in Bihar, India. The total number of affected people in Nepal is estimated to be 70,000. The flood has 
damaged around 6000 hectares of agricultural land (ICIMOD, 2008). The devastating flood of Koshi not only displaced public from their homes but destroyed the habitats of wildlife too. The flood displaced people, made encroachments in many parts of Koshi Tappu Wildlife Reserve disturbing both plants and animal. Very few migratory birds might have visited. Local birds, mammals, reptiles and fishes might have been swept away by the devastating flood. Many folds of wetlands have turned into sandy land, considering the heavy loss of habitats of several species of animals and plants. The need of assessing the impact of Koshi flood disaster 2008 on biodiversity of KTWR, CEPF and WWF Nepal has funded a small grant for the present study.

\section{Study area}

Koshi Tappu Wildlife Reserve extends between $86^{\circ} 55^{\prime}-87^{\circ} 05^{\prime} \mathrm{E}$ longitude and $26^{\circ} 34^{\prime}-$ $26^{\circ} 45^{\prime} \mathrm{N}$ latitudes covering an area of 17500 ha and buffer zone area covers 17300 ha. It includes part of Sunsari, Saptari and Udayapur districts of the eastern development region. Eastern and western embankments of 5-7 m high were constructed by the Koshi dam project to control flood. On the south of the reserve is a large expanse of open water, marshes and reed-beds, created by the construction of Koshi barrage between 1958 and 1964. Rectangular in shape, Koshi Tappu Wildlife Reserve is 16.3 $\mathrm{km}$ long and $9.3 \mathrm{~km}$ wide running along the Koshi river (Fig. 1). The area lies between 75 to $100 \mathrm{~m}$ altitudes above mean sea level. Saccharum and Typha are the dominant vegetation covering $80 \%$ of the KTWR area. Mixed forests of Dalbergia, Bombax and Acacia make up the remainder (Sah, 1997). The climatic condition of this area is tropical monsoonal type and experiences three distinct seasons summer (February to May), rainy (June-September) and winter (October-January). Koshi Tappu Wildlife Reserve lies in a low-lying area and its alluvial deposits are mainly composed of thin fine sand, silt and clay.

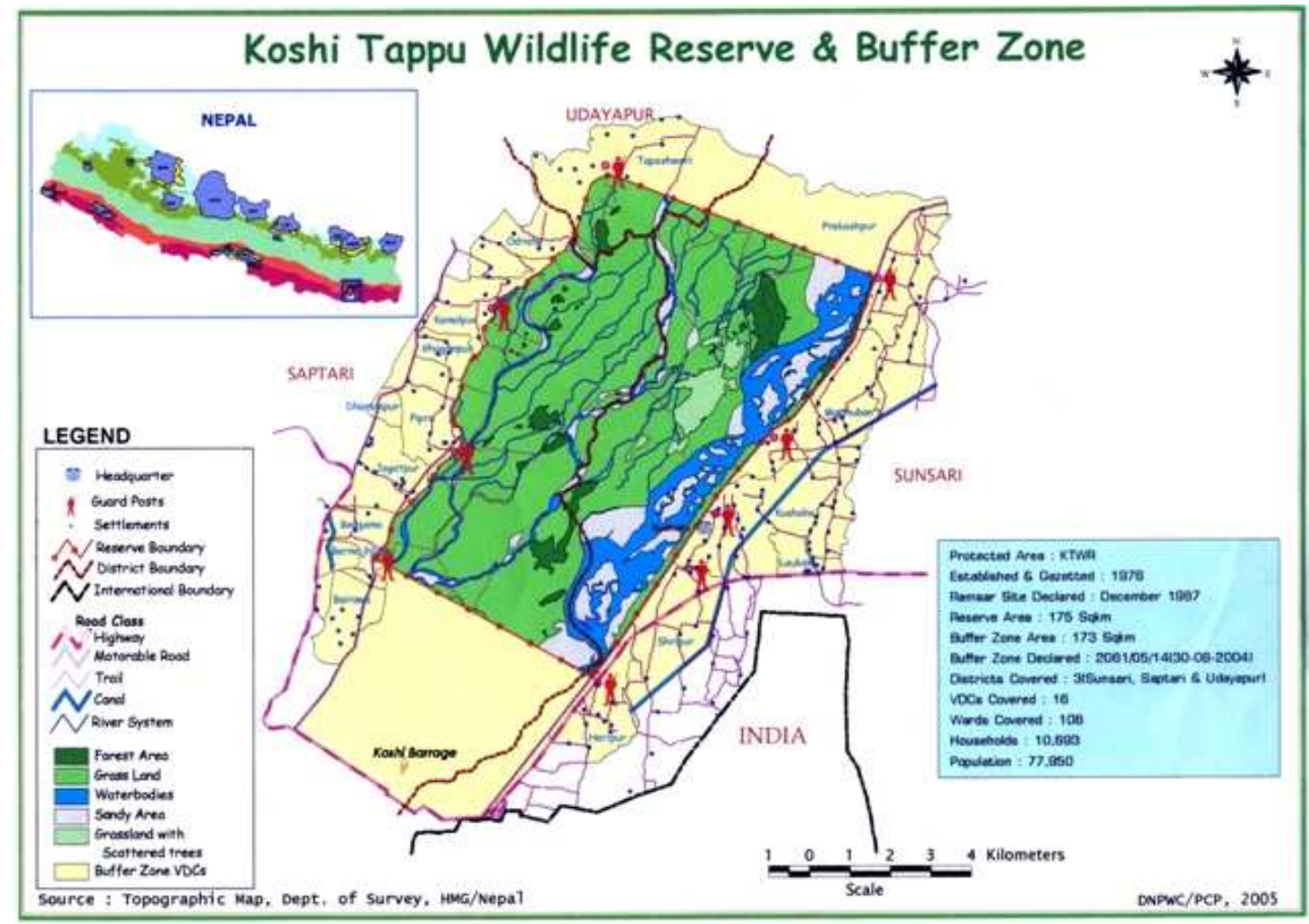

Figure 1. Map of Koshi Tappu Wildlife Reserve. 


\section{Methodology}

Access of different places within the reserve for surveying was made by the use of boats. Photographs and video records of degraded habitat and key faunas were also captured.

\section{Avifauna survey}

Bird surveys was done in morning and evening. Five 500-metres transects were set across survey sites (Sites 1,2,3,4 \& 5) as representatives of different habitats within Koshi Tappu Wildlife Reserve. The point count method was used at each transect (Table 1). At each of five points along the transects, all birds seen or heard within a 50 metre radius of the point, were recorded over ten minute intervals. Opportunistic sightings were recorded during the four days of the survey. The field books of Ali and Ripley (1986), Fleming et al. (2000) and Grimmett et al. (2000) were used in the field for bird identification. Avifaunal survey data was finally analyzed using ShannonWiener diversity index.

Table 1. Transect detail by survey site

\begin{tabular}{|c|c|c|c|c|c|c|}
\hline \multirow{2}{*}{ Transect } & \multicolumn{5}{|c|}{ Point co-ordinates } & \multirow{2}{*}{$\begin{array}{l}\text { Survey site } \\
\text { description }\end{array}$} \\
\hline & $\mathbf{I}$ & II & III & IV & $\mathbf{V}$ & \\
\hline \multirow[t]{2}{*}{1} & $26^{\circ} 31^{\prime} 17^{\prime \prime} \mathrm{N}$ & $26^{\circ} 31^{\prime} 16.7^{\prime \prime} \mathrm{N}$ & $26^{\circ} 31^{\prime} 16.4^{\prime \prime} \mathrm{N}$ & $26^{\circ} 31^{\prime} 16.1^{\prime \prime} \mathrm{N}$ & $26^{\circ} 31^{\prime} 15.8^{\prime \prime} \mathrm{N}$ & East of Koshi \\
\hline & $86^{\circ} 57^{\prime} 06.8^{\prime \prime} \mathrm{E}$ & $86^{\circ} 57^{\prime} 02.5^{\prime \prime} \mathrm{E}$ & $86^{\circ} 56^{\prime} 58.1$ 'E & $86^{\circ} 56^{\prime} 53.99^{\prime \prime} \mathrm{E}$ & $86^{\circ} 56^{\prime} 50^{\prime \prime} \mathrm{E}$ & barrage wetland area \\
\hline \multirow[t]{2}{*}{2} & $26^{\circ} 37^{\prime} 25.6^{\prime \prime} \mathrm{N}$ & $26^{\circ} 37^{\prime} 28.4^{\prime \prime} \mathrm{N}$ & $26^{\circ} 37^{\prime} 31.2^{\prime \prime} \mathrm{N}$ & $26^{\circ} 37^{\prime} 34.4 ” \mathrm{~N}$ & $26^{\circ} 37^{\prime} 37.6^{\prime \prime} \mathrm{N}$ & West of Koshi river \\
\hline & $87^{\circ} 01^{\prime} 46.2^{\prime \prime} \mathrm{E}$ & $87^{\circ} 01^{\prime} 48.2^{\prime \prime} \mathrm{E}$ & $87^{\circ} 01^{\prime} 50.4^{\prime \prime} \mathrm{E}$ & $87^{\circ} 01^{\prime} 52.7^{\prime \prime} \mathrm{E}$ & $87^{\circ} 01^{\prime} 55.1^{\prime \prime} \mathrm{E}$ & near Hattisar \\
\hline \multirow[t]{2}{*}{3} & $26^{\circ} 38^{\prime} 51.9^{\prime \prime} \mathrm{N}$ & $26^{\circ} 38^{\prime} 54.6^{\prime \prime} \mathrm{N}$ & $26^{\circ} 38^{\prime} 56.8^{\prime \prime} \mathrm{N}$ & $26^{\circ} 38^{\prime} 59.4^{\prime \prime} \mathrm{N}$ & $26^{\circ} 38^{\prime} 02^{\prime \prime} \mathrm{N}$ & West of Koshi river, \\
\hline & 8703'03.9'Е & $87^{\circ} 03$ '02.3'Е & $87^{\circ} 03^{\prime} 01.8 ' \mathrm{E}$ & $87^{\circ} 03^{\prime} 01.9$ 'Е & 8703'03.7'”E & North of Madhuvan \\
\hline \multirow[t]{2}{*}{4} & $26^{\circ} 37^{\prime} 51.7^{\prime \prime} \mathrm{N}$ & $26^{\circ} 38^{\prime} 22.9^{\prime \prime} \mathrm{N}$ & $26^{\circ} 38^{\prime} 17^{\prime \prime} \mathrm{N}$ & $26^{\circ} 38^{\prime} 14.2^{\prime \prime} \mathrm{N}$ & $26^{\circ} 38^{\prime} 12^{\prime \prime} \mathrm{N}$ & Pathari Army post \\
\hline & $87^{\circ} 45^{\prime} 38.3^{\prime \prime} \mathrm{E}$ & $86^{\circ} 56^{\prime} 40.2^{\prime \prime} \mathrm{E}$ & 8656’39.9’'E & $86^{\circ} 56^{\prime} 38.4$ 'Е & $86^{\circ} 56^{\prime} 37.4 ’ \mathrm{E}$ & grassland \\
\hline \multirow[t]{2}{*}{5} & $26^{\circ} 32^{\prime} 51.3^{\prime \prime} \mathrm{N}$ & $26^{\circ} 32^{\prime} 47.8^{\prime \prime} \mathrm{N}$ & $26^{\circ} 32^{\prime} 45.6^{\prime \prime} \mathrm{N}$ & $26^{\circ} 32^{\prime} 42.9^{\prime \prime} \mathrm{N}$ & $26^{\circ} 32^{\prime} 40.8^{\prime \prime} \mathrm{N}$ & North of Bardhaha \\
\hline & $86^{\circ} 53^{\prime} 47.4^{\prime \prime} \mathrm{E}$ & 865' 50.9'Е & $86^{\circ} 53^{\prime} 54^{\prime \prime} \mathrm{E}$ & $86^{\circ} 53^{\prime} 58.3^{\prime \prime} \mathrm{E}$ & 8654'01.4’'E & Wetland \\
\hline
\end{tabular}

\section{Dolphin survey}

Qualitative information on the abundance and distribution of dolphin were obtained from key informants: reserve personnel, naturalist, local people and indigenous people by informal interviews. Field surveys were done on dolphin potential sites (Chaudhary, 2007) (a) Koshi barrage (b) Koshi river near reserve headquarter and (c) Rajbas by using boat and population survey was done following WWF Nepal Program (2006). The census was conducted in the co-ordination of expert team of Nature Conservation and Health Care Council, Biratnagar in collaboration with experts of KTWR office.

\section{Wild buffalo survey}

Observation on wild buffalo was made taking necessary assistance of experts in the field.

\section{Monitoring of exotic fish through checklist}

Monitoring of exotic fish species within KTWR area was carried out by making checklist of all the fish available. Fish collection with the help of local fishermen using different types of gears such as cast net, gill net, scoop net, hook etc. was done. Fishes were preserved in suitable percentage of formalin in fresh condition for taxonomic identification and database. Identification was done following standard taxonomic books: Shrestha (1981, 1994), Shrestha (1990), Talwar and Jhingran (1991) and Jayaram (1999). 


\section{Habitat survey}

Different affected habitats like swampy area, marshy area of Koshi Tappu Wildlife Reserves due to Koshi flood were surveyed and observed.

\section{Wetland destruction survey}

An estimation of wetland destroyed by Koshi flood disaster 2008 within the reserve was done out by field survey method. Study of different wetlands area was made by collecting data of wetland damaged. GPS mapping method was used to prepare a map of wetland damaged.

\section{Results}

\section{Bird survey}

During observations of birds from $16^{\text {th }}$ April, 2009 to $15^{\text {th }}$ December, 2009 inside and in the periphery of Koshi Tappu Wildlife Reserve on regular basis, 120 species of birds belonging to 44 families and 103 genera were sighted. We observed 27 species as winter visitor, 4 species as summer visitor and 89 species as resident birds (Table 2). We calculated Shannon-Wiener Index value $(\bar{H})$ to be 5.256 .

Table 2. Checklist of birds based on several observations from $16^{\text {th }}$ April, 2009 to $15^{\text {th }}$ December, 2009

\begin{tabular}{|c|c|c|c|c|}
\hline SN & Common name & Scientific name & Family & Status \\
\hline$\overline{1}$ & Crested Serpent Eagle & Spilornis cheela & Accipitridae & Resident \\
\hline 2 & Osprey & Pandion haliaetus & Accipitridae & Winter visitor \\
\hline 3 & Pallas's Fish Eagle & Haliaeetus albicilla & Accipitridae & Winter visitor \\
\hline 4 & Shikra & Accipiter badius & Accipitridae & Resident \\
\hline 5 & White-rumped Vulture & Gyps bengalensis & Accipitridae & Resident \\
\hline 6 & Oriental Skylark & Alauda gulgula & Alaudidae & Resident \\
\hline 7 & Short-toed Lark & Calandrella cinerea & Alaudidae & Resident \\
\hline 8 & Bar-headed Goose & Anser indicus & Anatidae & Winter visitor \\
\hline 9 & Common Merganser & Mergus merganser & Anatidae & Winter visitor \\
\hline 10 & Common Pochard & Aythya ferina & Anatidae & Winter visitor \\
\hline 11 & Cotton Teal & Nettapus coromandelianus & Anatidae & Winter visitor \\
\hline 12 & Mallard & Anad platyrhynchos & Anatidae & Winter visitor \\
\hline 13 & Ruddy Shelduck & Tadorna ferruginea & Anatidae & Winter visitor \\
\hline 14 & Darter & Anhinga melanogaster & Anhingidae & Resident \\
\hline 15 & Cattle Egret & Bubulcus ibis & Ardeidae & Resident \\
\hline 16 & Black Bittern & Dupetor flavicollis & Ardeidae & Rare resident \\
\hline 17 & Grey Heron & Ardea cinerea & Ardeidae & Resident \\
\hline 18 & Intermediate Egret & Mesophoyx intermedia & Ardeidae & Resident \\
\hline 19 & Little Heron & Butorides striatus & Ardeidae & Resident \\
\hline 20 & Pond Heron & Adreola grayii & Ardeidae & Resident \\
\hline 21 & Purple Heron & Ardea purpurea & Ardeidae & Resident \\
\hline 22 & Yellow Bittern & Ixobrychus cinnamomeus & Ardeidae & Summer visitor \\
\hline 23 & Crow Pheasant & Centropus sinensis & Centropodidae & Resident \\
\hline 24 & Small Pied Kingfisher & Ceryle rudis & Cerylidae & Resident \\
\hline 25 & Eurasian Curlew & Numenius arquata & Charadriidae & Winter visitor \\
\hline 26 & Little-ringed Plover & Charadrius dubius & Charadriidae & Resident \\
\hline 27 & Northern Lapwing & Vanellus vanellus & Charadriidae & Winter visitor \\
\hline 28 & Red-wattled Lapwing & Vanellus indicus & Charadriidae & Resident \\
\hline 29 & Yellow-wattled Lapwing & Vanellus malabaricus & Charadriidae & Winter visitor \\
\hline 30 & Lesser Adjutant Stork & Leptoptilos javanicus & Ciconiidae & Resident \\
\hline 31 & Black Stork & Ciconia nigra & Ciconiidae & Winter visitor \\
\hline 32 & Black-necked Stork & Ephippiorhynchus asiaticus & Ciconiidae & Winter visitor \\
\hline 33 & Asian Openbill & Anastomus oscitans & Ciconiidae & Resident \\
\hline 34 & Plain Prinia & Prinia inornata & Cisticolidae & Resident \\
\hline 35 & Eurasian Collared Dove & Streptopelia decaocta & Columbidae & Resident \\
\hline 36 & Yellow-footed Green Pigeon & Treron phoenicoptera & Columbidae & Resident \\
\hline
\end{tabular}




\begin{tabular}{|c|c|c|c|c|}
\hline 37 & Spotted Dove & Streptopelia chinensis & Columbidae & Resident \\
\hline 38 & Wedge-tailed Green Pigeon & Treron sphenura & Columbidae & Resident \\
\hline 39 & Indian Roller & Coracias benghalensis & Coraciidae & Resident \\
\hline 40 & House Crow & Corvus splendens & Corvidae & Resident \\
\hline 41 & Indian Tree Pie & Dendrocitta vagabunda & Corvidae & Resident \\
\hline 42 & Jungle Crow & Corvus macrorhynchos & Corvidae & Resident \\
\hline 43 & Large Cuckooshrike & Coracina macei & Corvidae & Resident \\
\hline 44 & Common Hawk Cuckoo & Cuculus varius & Cuculidae & Resident \\
\hline 45 & Indian Cuckoo & Cuculus micropterus & Cuculidae & Resident \\
\hline 46 & Koel Cuckoo & Eudynamys scolopacea & Cuculidae & Resident \\
\hline 47 & Pied Cuckoo & Clamator jacobinus & Cuculidae & Summer visitor \\
\hline 48 & Lesser Whistling-duck & Dendrocygna javanica & Dendrocygnidae & Resident \\
\hline 49 & Ashy Drongo & Dicrurus leucophaeus & Dicruridae & Resident \\
\hline 50 & Black Drongo & Dicrurus adsimilis & Dicruridae & Resident \\
\hline 51 & Stork-billed Kingfisher & Pelargopsis capensis & Halcyonidae & Resident \\
\hline 52 & White-breasted Kingfisher & Halcyon smyrnensis & Halcyonidae & Resident \\
\hline 53 & Plain Martin & Riparia paludicola & Hirundinidae & Resident \\
\hline 54 & Common Iora & Aegithina tiphia & Irenidae & Resident \\
\hline 55 & Bronze-winged Jacana & Metopidius indicus & Jacanidae & Resident \\
\hline 56 & Pheasant-tailed Jacana & Hydrophasianus chirurgus & Jacanidae & Summer visitor \\
\hline 57 & Brown Shrike & Lanius cristatus & Laniidae & Winter visitor \\
\hline 58 & Grey-backed Shrike & Lanius tephronotus & Laniidae & Resident \\
\hline 59 & Long-tailed Shrike & Lanius schach & Laniidae & Resident \\
\hline 60 & Black-headed Gull & Larus ridibundus & Laridae & Winter visitor \\
\hline 61 & Caspian Tern & Sterna caspia & Laridae & Winter visitor \\
\hline 62 & Black-bellied Tern & Sterna acuticauda & Laridae & Resident \\
\hline 63 & Blue-throated Barbet & Megalaima asiatica & Megalaimidae & Resident \\
\hline 64 & Crimson-breasted Barbet & Megalaima haemacephala & Megalaimidae & Resident \\
\hline 65 & Blue-tailed Bee-eater & Merops philippinus & Meropidae & Resident \\
\hline 66 & Green Bee-eater & Merops orientalis & Meropidae & Resident \\
\hline 67 & Asian Paradise-flycatcher & Terpsiphone paradisi & Muscicapidae & Summer visitor \\
\hline 68 & Collared Bush Chat & Saxicola torquata & Muscicapidae & Resident \\
\hline 69 & Dusky Warbler & Phylloscopus fuscatus & Muscicapidae & Winter visitor \\
\hline 70 & Grey-headed Canary Flycatcher & Culicicapa ceylonensis & Muscicapidae & Winter visitor \\
\hline 71 & Oriental Magpie Robin & Copychus saularis & Muscicapidae & Resident \\
\hline 72 & Pied Bush Chat & Saxicola caprata & Muscicapidae & Resident \\
\hline 73 & Red-throated Flycatcher & Ficedula albicilla & Muscicapidae & Resident \\
\hline 74 & Verditer Flycatcher & Eumyias thalassina & Muscicapidae & Winter visitor \\
\hline 75 & White-breasted Fantail Flycatcher & Rhipidura aureola & Muscicapidae & Resident \\
\hline 76 & White-throated Fantail Flycatcher & Rhipidura albicollis & Muscicapidae & Resident \\
\hline 77 & Purple Sunbird & Nectarinia asiatica & Nectariniidae & Resident \\
\hline 78 & Black-hooded Oriole & Oriolus xanthornus & Oriolidae & Resident \\
\hline 79 & Black-napped Oriole & Oriolus chinensis & Oriolidae & Resident \\
\hline 80 & Golden Oriole & Oriolus oriolus & Oriolidae & Resident \\
\hline 81 & Citrine Wagtail & Motacilla citreola & Passeridae & Winter visitor \\
\hline 82 & House Sparrow & Passer domesticus & Passeridae & Resident \\
\hline 83 & Paddyfield Pipit & Anthus novaeseelandiae & Passeridae & Resident \\
\hline 84 & Pied Wagtail & Motacilla alba & Passeridae & Resident \\
\hline 85 & White-browned Wagtail & Motacilla maderaspatensis & Passeridae & Resident \\
\hline 86 & Yellow Wagtail & Motacilla flava & Passeridae & Winter visitor \\
\hline 87 & Large Cormorant & Phalacrocorax carbo & Phalacrocoracidae & Resident \\
\hline 88 & Little Cormorant & Phalacrocorax niger & Phalacrocoracidae & Resident \\
\hline 89 & Swamp Francolin & Francolinus gularis & Phasianidae & Resident \\
\hline 90 & Fulvous-breasted Woodpecker & Dendrocopos macei & Picidae & Resident \\
\hline 91 & Lesser Golden-backed Woodpecker & Dinopium benghalense & Picidae & Resident \\
\hline 92 & Streak-throated Woodpecker & Picus xanthopygaeus & Picidae & Resident \\
\hline 93 & Scaly-breasted Munia & Lonchura punctulata & Ploceidae & Resident \\
\hline 94 & Little Grebe & Tachybaptus ruficollis & Podicipedidae & Winter visitor \\
\hline 95 & Great Crested Grebe & Podiceps cristatus & Podicipedidae & Winter visitor \\
\hline 96 & Rose-ringed Parakeet & Psittacula krameri & Psittacidae & Resident \\
\hline 97 & Red-vented Bulbul & Pyconotus cafer & Pycnonotidae & Resident \\
\hline 98 & Red-whiskered Bulbul & Pycnonotus jocosus & Pycnonotidae & Resident \\
\hline 99 & Common Coot & Fulica atra & Rallidae & Winter visitor \\
\hline 100 & Common Moorhen & Galinula chloropus & Rallidae & Resident \\
\hline 101 & Purple Swamphen & Porphyrio porphyrio & Rallidae & Resident \\
\hline 102 & White-breasted Waterhen & Amaurornis phoenicurus & Rallidae & Resident \\
\hline
\end{tabular}




\begin{tabular}{|c|c|c|c|c|}
\hline 103 & Common Sandpiper & Actitis hypoleucos & Scolopacidae & Winter visitor \\
\hline 104 & Marsh Sandpiper & Tringa stagnatilis & Scolopacidae & Winter visitor \\
\hline 105 & Spotted Owlet & Athene brama & Strigidae & Resident \\
\hline 106 & Common Myna & Acridotheres tristis & Sturnidae & Resident \\
\hline 107 & Grey-headed Myna & Sturnus malabaricus & Sturnidae & Resident \\
\hline 108 & Jungle Myna & Acridotheres fuscus & Sturnidae & Resident \\
\hline 109 & Pied Myna & Sturnus contra & Sturnidae & Resident \\
\hline 110 & Clamorous Reed Warbler & Acrocephalus strenoreus & Sylviidae & Winter visitor \\
\hline 111 & Dusky Warbler & Phylloscopus fuscatus & Sylviidae & Winter visitor \\
\hline 112 & Jungle Babbler & Turdoides striatus & Sylviidae & Resident \\
\hline 113 & Rusty-cheeked Scimitar Babbler & Pomatorhinus erythrogenys & Sylviidae & Resident \\
\hline 114 & Striated Babbler & Turdoides earlei & Sylviidae & Resident \\
\hline 115 & Tailor Bird & Orthotomus sutorius & Sylviidae & Resident \\
\hline 116 & Black Ibis & Pseudibis papillosa & Threskiornithidae & Resident \\
\hline 117 & White Ibis & Threskiornis melanocephalus & Threskiornithidae & Winter visitor \\
\hline 118 & Spotted Owlet & Athene brama & Tytonidae & Resident \\
\hline 119 & Hoopoe & Uриро ерорs & Upupidae & Resident \\
\hline 120 & Oriental White-eye & Zosterops palpebrosus & Zosteropidae & Resident \\
\hline
\end{tabular}

\section{Dolphin survey}

A direct sighting survey of dolphin conducted from Rajabas to Koshi barrage in November and December of 2009 made a total count of 11 dolphins (Fig. 2). The team observed 6 dolphins in the river section upstream of Koshi barrage to Rajabas (Fig. 3) and 5 dolphins on downstream of Koshi barrage (Table 3). But indirect sighting of 3 dolphins in the coffer dam area made by local people has not been included in total count.

Table 3. Number of dolphin sighted in survey trip

\begin{tabular}{llcc}
\hline SN & Name of site & GPS co-ordinates & No. of dolphin sited \\
\hline & Direct sighting & $26^{\circ} 43^{\prime} 10.9^{\prime \prime} \mathrm{N} ; 87^{\circ} 05^{\prime} 48.4^{\prime \prime} \mathrm{E}$ & 1 \\
1. & Rajabans & $26^{\circ} 41^{\prime} 41.1^{\prime \prime} \mathrm{N} ; 87^{\circ} 033^{\prime} 51.8^{\prime \prime} \mathrm{E}$ & 2 \\
2. & Prakaspur & $26^{\circ} 39^{\prime} 25.1^{\prime \prime} \mathrm{N} ; 87^{\circ} 02^{\prime} 35.7^{\prime \prime} \mathrm{E}$ & 1 \\
3. & Near Vulture nest area & $26^{\circ} 36^{\prime} 17.8^{\prime \prime} \mathrm{N} ; 87^{\circ} 00^{\prime} 59.7^{\prime \prime} \mathrm{E}$ & 1 \\
4. & Katan area & $26^{\circ} 34^{\prime} 18.6^{\prime \prime} \mathrm{N} ; 86^{\circ} 57^{\prime} 7.5^{\prime \prime} \mathrm{E}$ & 1 \\
5. & Trijuga Dobhan & $26^{\circ} 31^{\prime} 26.3^{\prime \prime} \mathrm{N} ; 86^{\circ} 55^{\prime} 49.8^{\prime \prime} \mathrm{E}$ & 5 \\
6. & Total \\
\hline \multicolumn{2}{c}{ Koshi Barrage } & $26^{\circ} 38^{\prime} 51.9^{\prime \prime} \mathrm{N} ; 87^{\circ} 03^{\prime} 03.9^{\prime \prime} \mathrm{E}$ \\
\hline
\end{tabular}

\section{Number of dolphin}

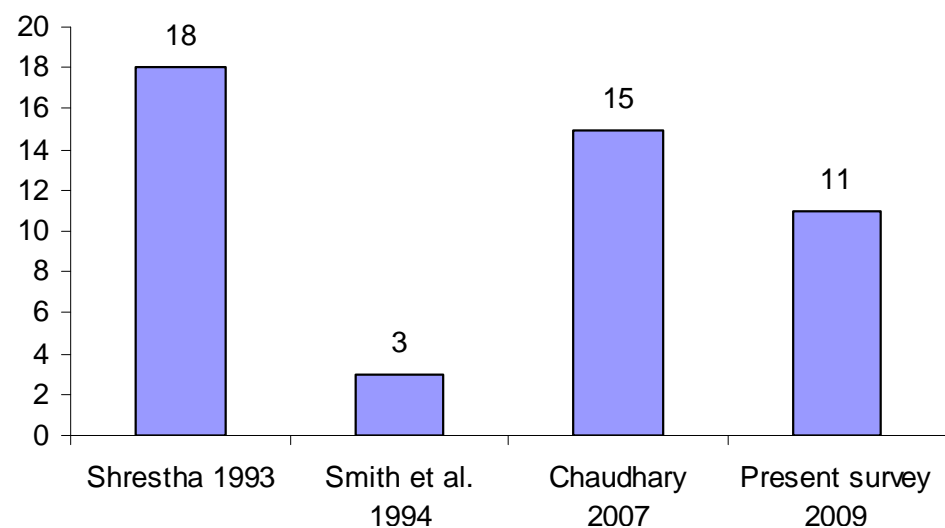

Figure 2. Population of dolphin in Koshi river from 1993 to 2009 carried out by different researchers 


\section{Number of dolphin}

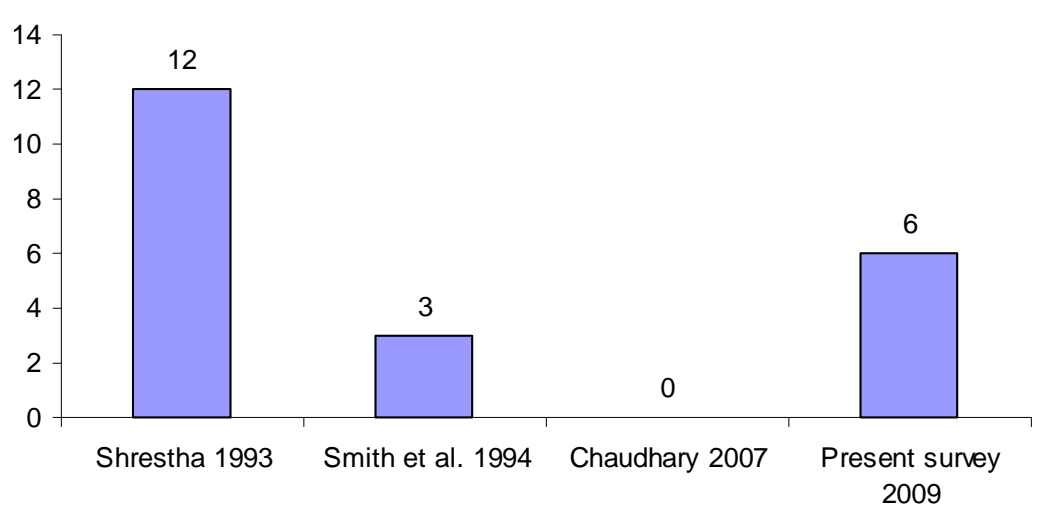

Figure 3. Population of dolphin in river section north of Koshi Barrage

\section{Wild water buffalo survey}

Regarding the census of wild water buffalo, Nilamber Mishra, Conservation Officer of KTWR suggested that it would be futile to re-census the wild water buffalo within short period as it was already done by joint venture of KTWR office and Conservation and sustainable use of wetland resources in Nepal (CSUWN). 219 wild water buffalo were counted on 3 days by KTWR office and CSUWN (Tables $4 \& 5$ ). The population structure of wild water buffalo in KTWR in 1976 (Dahmer, 1978), 1987, 1988 (Heinen, 2001) and 2009 (KTWR office, 2009) was shown in Table 6. We observed large herd of wild water buffalo many times in the west of KTWR office across the Koshi river in grassland areas.

Table 4. Population status of wild water buffalo

\begin{tabular}{|c|c|c|c|c|c|c|}
\hline Adult male & Adult female & Sub adult & $2^{\text {nd }}$ year calves & $1^{\text {st }}$ year calves & Back crossed & Total \\
\hline 34 & 101 & 39 & 22 & 23 & 74 & 219 \\
\hline
\end{tabular}

Source: KTWR office

Table 5. Blockwise population status of Wild water buffalo

\begin{tabular}{lllll}
\hline West of Koshi river & & East of Koshi river & & Total \\
\hline Block A & Block B & Block C & Block D & \\
South to Pathari post & North to Pathari post & South to Hawa mahal & North to Hawa mahal & \\
\hline 24 & 9 & 99 & 87 & 219 \\
\hline
\end{tabular}

Source: KTWR office

Table 6. Population structure of wild water buffalo in KTWR

\begin{tabular}{lcccccc}
\hline \multirow{2}{*}{ Year } & \multicolumn{2}{c}{ Adults } & \multirow{2}{*}{ Sub adult } & $\mathbf{2}^{\text {nd }}$ year & \multirow{2}{*}{$\mathbf{1}^{\text {st }}$ year } & \multirow{2}{*}{ Total } \\
\cline { 2 - 6 } & Male & Female & & & \\
\hline 1976 (Dahmer, 1978) & 12 & 18 & - & 22 & 11 & 63 \\
1987 (Heinen, 1993) & 32 & 29 & - & 14 & 16 & 91 \\
1988 (Heinen, 1993) & 37 & 33 & - & 8 & 15 & 93 \\
2000 (Heinen, 2000) & 56 & 53 & - & 17 & 19 & 145 \\
2009 (KTWR office) & 34 & 101 & 39 & 22 & 23 & 219 \\
\hline
\end{tabular}

\section{Fish survey}

Our regular collection of fish could enlist 64 species of fishes belonging to 15 families and 40 genera (Table 7). It included 44 common fishes, 6 fairly common, 2 less common, 3 most common, 9 uncommon fishes. 17 species of previously available fishes were not found during fish survey (Table 8). 
Table 7. Checklist of fish

\begin{tabular}{|c|c|c|c|}
\hline SN & Zoological name & Family & Status \\
\hline 1 & Colisa fasciatus & Anabantidae & Common \\
\hline 2 & Anabas testudineus & Anabantidae & Common \\
\hline 3 & Anguilla bengalensis & Anguillidae & Uncommon \\
\hline 4 & Mystus aor & Bagridae & Common \\
\hline 5 & M. cavasius & Bagridae & Common \\
\hline 6 & M. tengra & Bagridae & Common \\
\hline 7 & M. bheekerie & Bagridae & Less common \\
\hline 8 & M. vittatus & Bagridae & Less common \\
\hline 9 & Bagarius bagarius & Bagridae & Common \\
\hline 10 & Batasio batasio & Bagridae & Uncommon \\
\hline 11 & Xenentodon cancila & Belonidae & Common \\
\hline 12 & Chanda nama & Centropomidae & Common \\
\hline 13 & C. ranga & Centropomidae & Common \\
\hline 14 & C. gachua & Channidae & Common \\
\hline 15 & C. punctatus & Channidae & Common \\
\hline 16 & C. striatus & Channidae & Common \\
\hline 17 & C.morulius & Channidae & Uncommon \\
\hline 18 & Clarias batrachus & Clariidae & Common \\
\hline 19 & Semiplotus gongota & Cobitidae & Common \\
\hline 20 & Botia lohachata & Cobitidae & Fairly common \\
\hline 21 & B. dario & Cobitidae & Common \\
\hline 22 & Lepidocephalichthys guntea & Cobitidae & Common \\
\hline 23 & Nemacheilus botia & Cobitidae & Common \\
\hline 24 & Somileptes gongata & Cobitidae & Common \\
\hline 25 & Amblyphoryngodon mola & Cyprinidae & Common \\
\hline 26 & Aspidoparia jaya & Cyprinidae & Most common \\
\hline 27 & A. morar & Cyprinidae & Most common \\
\hline 28 & Barilius bola & Cyprinidae & Uncommon \\
\hline 29 & B. barana & Cyprinidae & Fairly common \\
\hline 30 & B. bendelisis & Cyprinidae & Common \\
\hline 31 & Chagunius chagunius & Cyprinidae & Fairly common \\
\hline 32 & Chela laubuca & Cyprinidae & Common \\
\hline 33 & Cirrhinus mrigala & Cyprinidae & Fairly common \\
\hline 34 & C. reba & Cyprinidae & Fairly common \\
\hline 35 & Danio devario & Cyprinidae & Common \\
\hline 36 & D. rerio & Cyprinidae & Common \\
\hline 37 & Esomuns dandricus & Cyprinidae & Common \\
\hline 38 & Garra lamta & Cyprinidae & Common \\
\hline 39 & Labeo rohita & Cyprinidae & Common \\
\hline 40 & Labeo calbasu & Cyprinidae & Common \\
\hline 41 & Puntius sophore & Cyprinidae & Most common \\
\hline 42 & P. sarana & Cyprinidae & Common \\
\hline 43 & P. ticto & Cyprinidae & Uncommon \\
\hline 44 & P. conchonius & Cyprinidae & Uncommon \\
\hline 45 & Tor putitora & Cyprinidae & Uncommon \\
\hline 46 & Crossocheilus latius & Cyprinidae & Common \\
\hline 47 & Glossogobius giuris & Gobiidae & Common \\
\hline 48 & Macrognathus aculeatus & Mastacembelidae & Common \\
\hline 49 & Mastacembelus armatus & Mastacembelidae & Common \\
\hline 50 & Heteropneustes fossilis & Saccobranchidae & common \\
\hline 51 & Ailia coila & Schilbeidae & Common \\
\hline 52 & Clupisoma garua & Schilbeidae & Common \\
\hline
\end{tabular}




\begin{tabular}{llll}
\hline 53 & Eutropiichthys vacha & Schilbeidae & Common \\
54 & Pseudeutropius atherinoides & Schilbeidae & Common \\
55 & P. murius batarensis & Schilbeidae & Common \\
56 & Ompok bimaculatus & Siluridae & Common \\
57 & Wallago attu & Siluridae & Common \\
58 & Bagarius bagarius & Sisoridae & Uncommon \\
59 & Gagata cenia & Sisoridae & Common \\
60 & G. viridescens & Sisoridae & Common \\
61 & Glyptothorax cavia & Sisoridae & Common \\
62 & G. pectinopterus & Sisoridae & Common \\
63 & G. telchitta & Sisoridae & Uncommon \\
64 & G. trilineatus & Sisoridae & Fairly common \\
\hline
\end{tabular}

Table 8. Unavailable fishes during collection

\begin{tabular}{lll}
\hline SN & Zoological name & Family \\
\hline 1 & Amblycep mongois & Amblycipitidae \\
2 & Rita rita & Bagridae \\
3 & Chaca chaca & Chacidae \\
4 & Gadusia godanahiai & Clupeidae \\
5 & G. chapra & Clupeidae \\
6 & Achanthocephala pangia & Cobitidae \\
7 & Puntius phutunis & Cyprinidae \\
8 & P. chola & Cyprinidae \\
9 & P. gelius & Cyprinidae \\
10 & Oxygaster bacaila & Cyprinidae \\
11 & Barilius jalkapoorie & Cyprinidae \\
12 & Septipina phasa & Engraulidae \\
13 & Nandus nandus & Nandidae \\
14 & Notopterus chitala & Notopteridae \\
15 & Erethistes pussilus & Sisoridae \\
16 & Siror rhabdophorus & Sitoridae \\
17 & Tetradon cutcutia & Tetradontidae \\
\hline
\end{tabular}

Habitat and wetland destruction

Important resting areas of residential and migratory birds like Titirgachi Daha has dried due to Koshi flood. More than $75 \%$ Swamp Partridge habitat in the eastern dam site was found to have lost due to dam and spur construction activities and flood displaced people. We estimated 113 ha area (Fig. 4) in eastern embankment of KTWR as most disturbed area due to construction activities for making spur, large number of heavy vehicles transportation and use of steamer in the Koshi river.

\section{Discussion}

We observed 120 species, 42 genera of 25 families of birds. Bird species availability in the present environment of the reserve and its surroundings depicts that there is every possibility of rehabilitation of bird diversity in the future through proper management. Both migratory and resident birds were impacted greatly by the flood. In 2008 few migratory birds such as Ruddy Shelduck, Common Pochard turned out for short time then left the place for another safe area. It might happen so because of disturbance and unavailability of food. A few representatives of some migratory birds such as Ruddy Shelduck, Bar-headed Goose, Mallard, Black Stork and Great Crested Grebe were observed. They were sighted along with resident birds such as Large 
Cormorant, Darter, Cattle Egret, Intermediate Egret and Pond Heron. So far the published Checklists of birds (Baral, 2000; Grimmett et al., 2000; Baral \& Inskipp, 2001; 2004; Baral, 2005; Bird Conservation Nepal, 2006; Thapa Chhetry, 2008) prepared in different months make it clear that some birds such as Eurasian Kingfisher, White-necked Stork, Collared Pygmy Owlet, either they have little scattered population so they didn't appear or they have left the area for the time being because of disturbances or food scarcity. Among the birds mentioned above, migratory ones are regular visitors of KTWR in the past. They were sighted in the month of November every year in the past. Among the migratory birds sighted in the month of November and upto the second week of December 2009, the number of Ruddy Shelduck was praiseworthy among aquatic water fowls. Its population was remarkably the highest. Common Pochard, Mallard, Bar-headed Goose, Black Stork, Common Merganser and Great crested Grebe had a few number of representatives. No doubt the population of every bird species is declining but at what ratio, it has to be estimated globally then only right status of each species of bird can be estimated. Annual Waterbird Counts highlighted the decline of winter visitor since 1999 in KTWR. A total of nearly 9800 birds were counted in February 2003 at the site in one day when more than 50000 birds were estimated in the past years (Choudhary, 2003). We counted not more than 2000 in the present survey. The population of globally threatened Lesser Adjutant has been declining every year in and around Koshi Tappu Wildlife Reserve (Baral, 2005). Pokharel (1998) recorded 65 individuals in Koshi Tappu during his study in 1994-1995. We observed not more than 20 individuals and no nest in and around Koshi Tappu Wildlife Reserve. Hunting and alteration of its habitat in Koshi Tappu area reduced its feeding area (Shakya, 1995; Giri, 1997; Petersson, 1998). Loss of Bombax ceiba is recognized as a major threat in Chitwan even inside a protected area (Gyawali, 2003) for large bird species. We observed White-rumped Vulture nests inside the reserve area in Bombax ceiba. At the same time, many trees of Bombax ceiba were found to be illegally felled near the area of vulture nest. KTWR holds the largest population of globally threatened Swamp Francolin in Nepal (Baral, 1998). Grasslands in the eastern embankments and spurs from reserve office to Madhuvan are the major habitat of Swamp Francolin in KTWR. These areas are disturbed by dam and spurs construction activities after Koshi Flood disaster. Grasslands turned into the barren land as for storage of stones and other equipments they were used. From the above observation it can be concluded that the migratory birds left the feeding ground of Koshi river within a week or so because of either disturbance created by fishing activities or lack of food. As a good number of fisherman belonging to flood displaced community have been given permission, so almost all places in the river, fishermen were seen fishing using gillnet, cast net and large net. Purple Gallinules are unsafe in the south, so they also have migrated towards north. Black-necked Stork, White-necked Stork, Painted Stork also were sighted in winter as well as in other seasons also in the past but they didn't turn out in our survey of $36 \mathrm{~km}$ from Rajbas to Koshi Barrage on boat. Resident birds such as Eurasian Kingfisher, Purple Swamphen and White-breasted Kingfisher were seen to have been disturbed by human activities because of flood disaster. The flood disaster has left a chronic problem for the Koshi Tappu Wildlife Reserve in addition to its number of unsolved problems. Shannon-Wiener diversity Index $(\bar{H})$ represents the uncertainty or information of a community. The more variable its composition, the more variable each sample of it would be. It varies from 0 , for a community of one species only, to values of 7 or more in rich biodiversity regions (Barbour et al., 1980). The higher the diversity index the greater the number of species and evenness of their populations 
(Bibby et al., 2000). Bird Shannon -Wiener diversity index in KTWR was calculated as 5.256 in the present survey which indicates KTWR still holds a good abundance of bird diversity even after flood. No other previous literature regarding the diversity index of Bird diversity in KTWR was found to compare the results.

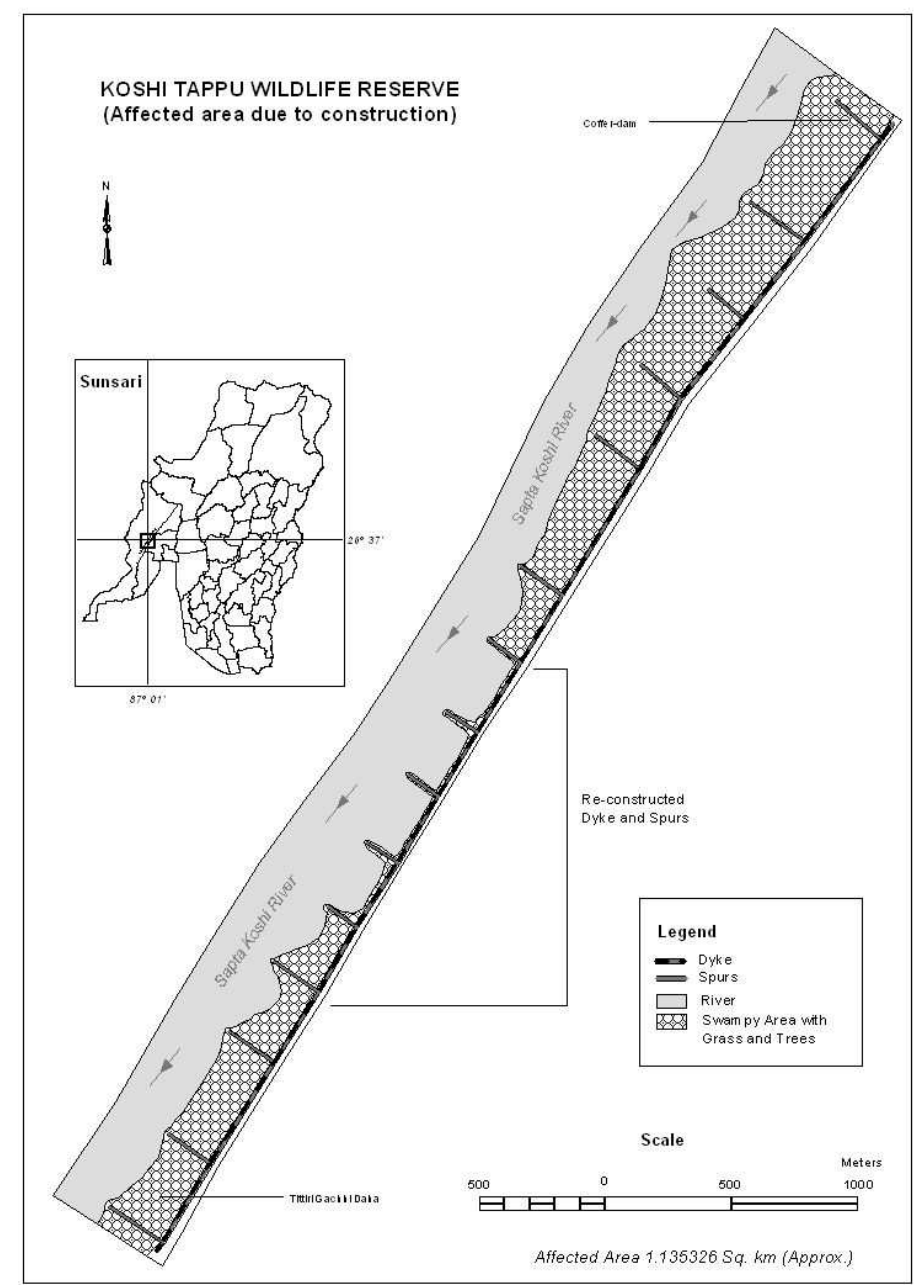

Figure 4. Affected area due to flood and construction activities within KTWR

Perhaps Dolphin census in the Koshi river course, starting from Rajabas to Koshi Barrage might be the first step of its kind. Gangetic Dolphin is the only big aquatic mammal found in rivers such as Koshi, Karnali and Narayani (Smith et al., 1994). Shrestha (1993) observed 3 individuals near Barahachhetra and Chatara, 6 individuals near Chatara, 3 individuals in Kusaha and 6 individuals in Koshi Barrage area and estimated the total population of 18 individuals in the Koshi river. Smith et al. (1994) surveyed the area between the confluence of the Arun and Sun Koshi rivers; and the Koshi Barrage and found only 3 dolphins. In the southern section of the barrage, Chaudhary (2007) estimated a population of 15 individuals distributed within the range of $2 \mathrm{~km}$ in the river section south of Koshi Barrage but no dolphins were sighted by Chaudhary (2007) in the river section north of Koshi Barrage. In our survey, the first census concluded with the sightings of 9 dolphins. Our survey estimated a total of 6 individuals in the river section north of Koshi Barrage upto Rajabas and 5 individuals in the downstream of Koshi Barrage. All these data indicated that no dolphins were observed north of Koshi Barrage upto Barahachhetra since 1994 to 2007. The Koshi Barrage blocked the migration of fish and aquatic animals causing shortage of food for dolphin in the river section north of Koshi 
Barrage (Shrestha, 1993). During high water periods, there is a chance of dolphins to move downstream through the Koshi Barrage but the high current might have prevented them from swimming upstream through the barrage. The effects of subdividing a single population into non-interacting insular units increase their vulnerability to environmental, demographic and genetic (Haque et al., 1998). Reappearance of dolphin in north of Koshi Barrage upto Rajabas in the present survey indicates that Koshi Flood disaster 2008 might be the reason of dolphin to move north of Koshi river as the river did not flow through the Koshi Barrage during flood period. But the use of Steamer after Koshi flood in the Koshi river was harmful for dolphins. Heavy boat traffic and overfishing were the causes of extinction of Dolphin Baiji in China.

The wild water buffalo in KTWR is highly endangered; with the few remaining populations already affected or likely to be affected by hybridization with domestic buffalo (Flamand et al., 2003). Population estimation of wild water buffalo in KTWR was initiated by Dahmer (1978) with a count of 63 individuals in 1976. Heinen (1993) observed total of 91 individuals in 1987 and 93 in 1988. In total, 145 wild water buffalo were censused in 2000 by Heinen (2001). Recent count of wild water buffalo by joint venture of KTWR office and CSUWN (2009) estimated a total count of 219. All these data show a population growth of wild water buffalo in KTWR is satisfactory. But 2 of the predefined as wild buffalo and 7 of the predefined as domestic buffalo showed evidence of mixed ancestry in the genetic analysis of wild water buffalo examined (Flamand et al., 2003). Habitat of wild water buffalo was also studied in our survey. All the locations where wild water buffalo live were visited taking the help of Ranger, Game scout and cowherd. The number has increased remarkably. In fact, the number of wild water buffalo was seen praiseworthy but whether they were of purebred wild or hybrid could not be ascertained. Ideally, in all cases, detailed genetic studies are further needed to identify the purebred wild. Inside the reserve, the habitat was seen not drastically altered; however, heavy grazing of grasslands shows that they shared food and other space with feral cattle. The uncontrolled illegal entry of domesticated cows and buffaloes may be one of the main reasons of declining number of wild water buffalo in the time to come.

A small population of Gangetic Dolphin found in Koshi river indicates that the status of fishes in the river is not so bad. Altogether 92 species of fishes was identified by Thapa Chhetry (2008) during the course of 3 years period. But we recorded 64 species in 8 months out of 117 species in KTWR (IUCN, 1998). Altogether 17 species of commonly available fish species of KTWR area could not be collected instead of regular collection. During fish collection, population was also estimated and was found the highest population of Aspidoparia morar and A. jaya. In the collection there was least number of representatives of the following fish species: Tor putitora, Bagarius bagarius, Barillius bola, Notopterus notopterus, Anguilla bengalensis. None of the cultivated exotic fish species was found in the collection. This trend of fish species availability in the Koshi river depicts that either Koshi flood disaster has impacted on the fish population which inhabit mainly in swamp land. In regard of Puntius species, it was found that only Puntius sophore has become dominated species. Some other species which were also found in KTWR showed least number of representatives.

In addition to flood impacts on habitat destruction, from all sides, herd of cattle and group of herd-men enter the reserve freely, this kind of activities are making the 
reserve totally public right property rather than a protected reserve semi-public right property.

\section{Acknowledgements}

We acknowledge our thanks to WWF Nepal and Critical Ecosystem Partnership Fund (CEPF) for small grant. Thanks are also due to several key informants, local people and boatmen of the study site for their active support in the field work.

\section{References}

Ali, S. \& S.D. Ripley. 1986. Handbook of the Birds of India and Pakistan, Vol. V. Oxford University Press.

Baral, H.S. 2000. Birds of Koshi. Bird Conservation Nepal. Pub. No.3, DNPWC, Kathmandu, Nepal.

Baral, H.S. \& C. Inskipp. 2001. Important bird areas in Nepal: A report to the Royal Society for the Protection of Birds, U.K. Kathmandu: Bird Conservation Nepal.

Baral, H.S. \& C. Inskipp. 2004. The state of Nepal's birds 2004. Kathmandu: Department of National Parks and Wildlife Conservation, Bird Conservation Nepal and IUCN Nepal.

Baral, H.S. 1998. Status, distribution and habitat preferences of Swamp Francolin Francolinus gularis in Nepal. Ibisbill 1: 35-70.

Baral, H.S. 2005. Surveys for Lesser Adjutant Leptoptilos javanicus in and around Koshi Tappu Wildlife Reserve, Nepal. Forktail 21: 190-193.

Baral, H.S. \& C. Inskipp. 2005. Important bird areas in Nepal: Key sites for conservation. Bird Conservation Nepal and Birdlife International, Kathmandu and Cambridge.

Barbour, M.G., J.H. Burk \& W.D. Pitts. 1980. Terrestrial plant ecology. The Benjamin/ Cummings Publishing Co., Inc., California.

Bibby, C., M. Jones \& S. Marsden. 2000. Expeditions field techniques: Bird surveys. Birdlife International, UK.

Bird Conservation Nepal. 2006. Birds of Nepal: An official checklist. Bird Conservation Nepal and Department of National Park and Wildlife Conservation, Kathmandu.

Chaudhary, H. 2003. One day bird survey at Koshi Tappu Wildlife Reserve. Danphe 12(1/2): 6 .

Chaudhary, S. 2007. Status of and threats to Ganges River Dolphin (Platanista gangetica) in Koshi river, Nepal. M.Sc. thesis, University of Klagenfurt, Austria.

Dahmer, T.D. 1978. Status and distribution of the wild Asian buffalo Bubalus bubalis in Nepal. MS thesis, University of Montana, Missouri, Montana.

Flamand, J.R.B., D. Vankan, K.P. Gairhe, H. Duong \& J.S.F. Barker. 2003. Genetic identification of wild Asian water buffalo in Nepal. Animal Conservation 6: 265270.

Fleming, R.L. (Sr.), R.L. (Jr.) Fleming \& L.S. Bangdel. 2000. Birds of Nepal with reference to Kashmir and Sikkim. First Adarsh Impression, Gaurav Offset, Delhi.

Giri, T. 1997. Habitat loss at Koshi. Danphe 6(2): 1.

Grimmett, R., C. Inskipp \& T. Inskipp. 2000. Birds of Nepal. Christopher Helm, A \& C Black, London.

Gyawali, N. 2003. Population status and habitat preference of Lesser Adjutant Leptoptilos javanicus in Royal Chitwan National Park, mid-lowland Nepal. Unpublished report submitted to Oriental Bird Club, U.K.

Haque, A.K.M., M.S. Hossain \& A. Khan. 1998. River dolphins in Bangladesh: Conservation and effects of water development. Environmental Management (22): 3, 323-335. 
Heinen, J.T. 1993. Population viability and management recommendations for wild water buffalo (Bubalus bubalis) in Kosi Tappu Wildlife Reserve, Nepal. Biological Conservation 65: 29-34.

Heinen, J.T. 2002. Phenotypic and behavioural characteristics used to identify wild buffalo (Bubalus bubalis) from feral backcrosses in Nepal. J. Bomb. Nat. Hist. Soc. 99(2): 173-183.

ICIMOD. 2008. Koshi flood disaster. Report prepared by ICIMOD, August 27.

IUCN. 1998. An interpretation and education system for Koshi Tappu Wildlife Reserve and its bufferzones. IUCN, Nepal.

Jayaram, K.C. 1999. The freshwater fishes of Indian region. Narendra Publishing House, Delhi, India.

Petersson, D. 1998. What is happening at Koshi Tappu Wildlife Reserve? Danphe 7(1/2): 6-7.

Pokharel, P. 1998. Food items and feeding behavior of the Lesser Adjutant Stork, Leptoptilos javanicus in the Koshi Tappu Wildlife Reserve. Ibisbill 1: 71-86.

Sah, J.P. 1997. Koshi Tappu Wetlands: Nepal's Ramsar site. IUCN, Nepal.

Shakya, S. 1995. Bird massacre in Nepal. Bird Conservation Nepal Bull. 4(3): 5.

Shrestha, J. 1981. Fishes of Nepal. Curriculum Development Centre, Tribhuvan University, Kathmandu, Nepal.

Shrestha, J. 1994. Fishes, fishing implements and methods of Nepal. (Smt. M.D. Gupta), Lalitpur colony, Lashkar (Gwalior) India.

Shrestha, T.K. 1990. Rare fishes of Himalayan waters of Nepal. J. Fish Biol. 37: 213-216.

Shrestha, T.K. 1993. Ecology, status appraisal, conservation and management of Gangetic Dolphin Platanista gangetica in the Koshi river of Nepal. J. Freshwater Biol. 5 (1): 93-105.

Smith, B.D., R.K. Sinha, U.R. Regmi \& K. Sapkota. 1994. Status of Ganges River Dolphin in Karnali, Mahakali, Narayani and Saptakosi rivers of Nepal and India. Vol. 10, Issue 3. Marine Mammal Science. Blackwell Synergy.

Talwar, P.K. \& A.G. Jhingran. 1991. Inland fishes of India and adjacent countries. Vol. I and II, Oxford and IBH Publishing Co. Pvt. Ltd, New Delhi, India.

Thapa Chhetry, D. 2008. Studies on physico-chemical parameter and macrobiota with special reference to fishes in the wetland of Koshi Tappu wildlife Reserve and its surroundings. Ph.D. thesis, North Bengal University, Darjeeling districts, Siliguri, West Bengal, India.

WWF. 2006. Status, distribution and conservation threats of Ganges River Dolphins in Karnali river, Nepal. Published by WWF, Kathmandu, Nepal. 